\title{
Red de comercialización y uso de plaguicidas en el partido de Tandil, sector del sudeste de la provincia de Buenos Aires, Argentina'
}

\author{
Paula Rocío Pena Gómez² \\ Universidad Nacional del Centro de la Provincia de Buenos Aires
}

@ [ paulapenadyga@gmail.com ]

Rocío Fernández San Juan ${ }^{3}$

Universidad Nacional del Centro de la Provincia de Buenos Aires Consejo Nacional de Investigaciones Científicas y Técnicas

@ [ mrociofsj@gmail.com ]

\section{Patricia Vázquez ${ }^{4}$}

Universidad Nacional del Centro de la Provincia de Buenos Aires Consejo Nacional de Investigaciones Científicas y Técnicas

@ [ patriciavazquez11@gmail.com ]

\section{Agustina Cortelezzi ${ }^{5}$}

Universidad Nacional del Centro de la Provincia de Buenos Aires Consejo Nacional de Investigaciones Científicas y Técnicas

@ [ aguscorte@gmail.com ]

RECIBIDO 11-03-2021

ACEPTADO 12-08-2021

Cita sugerida: Pena Gómez, P. R., Fernández San Juan, R., Vázquez, P. y Cortelezzi, A. (2021). Red de comercialización y uso de plaguicidas en el partido de Tandil, sector del sudeste de la provincia de Buenos Aires, Argentina. Revista Huellas, Volumen 25, № 2, Instituto de Geografía, EdUNLPam: Santa Rosa. Recuperado a partir de: http://cerac.unlpam.edu.ar/index.php/huellas

DOI: http://dx.doi.org/10.19137/huellas-2021-2522

\section{Resumen}

La actividad agrícola conforma un motor de la economía argentina, y ante la extensiva utilización de plaguicidas en la elaboración de alimentos, es fundamental comprender el manejo de los mismos para evaluar sus efectos sobre el ambiente y la salud de las personas. El objetivo fue conocer los principales aspectos de la red de actores que usan y comercializan plaguicidas en el par- 
tido de Tandil (Buenos Aires) durante 2019 y 2020. Se entrevistó a referentes calificados y se encuestó a productores agrícolas de la zona. Se identificaron 10 actores principales, agrupados en 3 categorías (organismos gubernamentales, asociaciones civiles y privados) los cuales demostraron encontrarse vinculados a través de actividades tales como control, capacitación o flujos comerciales. Los actores clave coincidieron en la escasez de recursos asignados al control, la baja utilización de la receta agronómica y el diverso destino final de los bidones vacíos. Sumado a esto, se evidencia la mirada antropocéntrica en relación a la naturaleza, la falta de asesoramiento profesional en los campos, y desconfianza en la producción agroecológica. Se concluye la necesidad de incorporar nuevos actores a la red, generar políticas públicas que posibiliten un mayor control, y la urgencia de transitar un cambio de paradigma productivo.

Palabras clave: red de actores, modelo productivo agroindustrial, escasez de control, falta de asesoramiento, gestión de políticas públicas, cambio de paradigma productivo, alimentos saludables

\title{
Marketing network and pesticide use in the district of Tandil, a sector in the southeast of the province of Buenos Aires, Argentina
}

\begin{abstract}
Agricultural activity is a driving force of the Argentine economy and, given the extensive use of pesticides in food production, it is essential to understand their handling to assess their impact on the environment and people's health. The aim of this work was to know the main aspects of the network of actors that use and commercialize pesticides in the Tandil district (province of Buenos Aires) during 2019 and 2020. Qualified actors from this field were interviewed and agricultural producers in the area were surveyed. Ten main actors were identified and grouped under three categories (government agencies, civil and private associations) which proved to be related to through activities such as control, training or trade flows. The key actors agreed on the scarce resources assigned to the control, the restricted use of the agronomic formula and the various final uses of the empty drums. In addition to this, the anthropocentric stance in relation to nature, the lack of professional counselling in the farms, and distrust in agroecological production became evident. It is concluded that it is necessary to incorporate new actors into the network, to generate public policies that allow for greater control, and to urgently produce a change in the productive paradigm.
\end{abstract}

Keywords: network of actors, agro-industrial production model, lack of control, lack of advice, management of public policies, change of production paradigm, healthy food

\section{Rede de comercialização e uso de pesticidas no partido de Tandil, setor sudeste da província de Buenos Aires, Argentina}

\section{Resumo}

As atividades agrícolas conformam o motor da economia argentina e perante a extensa utilização de pesticidas na elaboração de alimentos, é fundamental compreender o controle para avaliar seus efeitos sobre o ambiente e a saúde das pessoas. 0 objetivo foi conhecer os principais aspectos da rede de atores que usam e comercializam pesticidas na zona de Tandil (Buenos Aires) durante 2009 e 2010. Entrevistou-se a referentes qualificados e se realizaram enquetes 
a produtores agrícolas da zona. Identificaram-se 10 atores principais, agrupados em 3 categorias (organismos governamentais, associações civis e privadas) os quais demostraram encontrar-se vinculados a través de atividades tais como controle, capacitação ou fluxos comerciais. Os atores chaves coincidiram na escassez de recursos assignados ao controle, à baixa utilização da receita agronômica e o diverso destino final dos tambores vazios. Além disto, evidencia-se um olhar antropocêntrico em relação à natureza, à falta de assessoramento profissional nos campos e desconfiança na produção agroecológica. Conclui-se a necessidade de incorporar novos atores à rede, gerar políticas públicas que possibilitem um maior controle e a urgência de transitar uma mudança de paradigma produtivo.

Palavras-chave: Rede de atores; Modelo produtivo agroindustrial; Escassez de controle; Falta de assessoramento; Gestão de políticas públicas; Mudança de paradigma produtivo; Alimentos saudáveis

\section{Introducción}

La denominada revolución verde se basó en la selección genética de nuevas variedades de cultivo de alto rendimiento, gracias a la difusión de una agricultura asociada a la explotación intensiva permitida por insumos externos tales como agua de riego, fertilizantes, plaguicidas y maquinaria pesada (Ceccon, 2008). Pese a que dicho fenómeno se remonta a las décadas del cincuenta y sesenta, los profundos cambios provocados en los sectores agropecuarios constituyen hasta el día de hoy un foco de degradación ambiental, generando un punto de inflexión a partir del cual la agricultura comienza a hacer hincapié en el aumento de la rentabilidad (Segrelles, 2001). Posteriormente, la incorporación de la siembra directa como práctica conservacionista y su paquete tecnológico asociado, profundizaron un modelo basado en la agriculturización (Pordomingo, 2003), entendiendo dicho proceso como el avance progresivo e ininterrumpido de las tierras para cultivos agrícolas, en detrimento de aquellas destinadas a usos ganaderos o mixtos (Manuel-Navarrete et al., 2005). De acuerdo a lo expresado por Sili (2005), la agriculturización no sólo conlleva un cambio de actividades, sino que se observa también una transformación del sistema natural, el paisaje, y el territorio, junto con las relaciones sociales que en él se gestan. Dicho territorio se entiende como las relaciones de poder y sentido que diversos actores le asignan a un espacio socialmente construido (Albaladejo et al., 2013). El mencionado proceso implica una mayor rentabilidad en comparación con otras producciones y ausencia de mecanismos estatales equilibradores o de 
compensación, lo que explica su vertiginosa expansión (Rodríguez, 2012). Este crecimiento estuvo acompañado de un divorcio entre los principios ecológicos y los principios productivistas de los establecimientos rurales (Sarandón y Flores, 2014), poniendo en riesgo tanto la calidad del ambiente como su capacidad productiva.

Los procesos agrícolas anteriormente mencionados se asocian a una serie de problemas ambientales que ponen en duda su permanencia en el tiempo al no considerar las externalidades negativas producidas (Carrasco et. al., 2012), entre ellos, la modificación del hábitat (lo que conlleva a la introducción de especies invasoras, la erosión y la pérdida de los suelos), la contaminación de los ecosistemas con plaguicidas y fertilizantes, y la afectación de la salud de la población humana expuesta al contacto y riesgo del consumo de agua y alimentos contaminados con agentes químicos (Vázquez y Zulaica, 2013). En relación al uso de plaguicidas, es importante resaltar que los datos disponibles sobre la comercialización y facturación de estos insumos en el país son los que brinda la Cámara de Sanidad Agropecuaria y Fertilizantes. Según este organismo, se detecta un aumento en el volumen de producto fitosanitario vendido del $13 \%$ respecto al año anterior (CASAFE, 2016). El partido de Tandil (provincia de Buenos Aires), no queda exento al creciente proceso de agriculturización (Vázquez et al. 2019, Somoza et al. 2020). La ausencia de información ligada a los plaguicidas utilizados, sumado a la falta de datos relacionados a la dinámica de comercialización y aplicación de los mismos, han motivado la presente investigación. Por lo tanto, nuestro objetivo fue conocer los principales aspectos de la red de actores que usan y comercializan plaguicidas en el partido de Tandil durante el período comprendido entre los años 2019 y 2020.

\section{Área de estudio y metodología}

El partido de Tandil, se encuentra en el sudeste de la provincia de Buenos Aires, Argentina (Fig. 1). La población rural del mismo incluye a las localidades de Gardey y María Ignacia Vela, así como los parajes De la Canal, La Pastora, Fulton, Azucena, Desvío Aguirre e Iraola. La ciudad de Tandil, cabecera del partido, se encuentra a unos $350 \mathrm{~km}$ de la ciudad de Buenos Aires, vinculándose con otras localidades de la provincia a través de la RN 226, la RP 30 y la RP 74.

De acuerdo a los datos proporcionados por el INDEC a partir del último Censo Nacional de Población y Vivienda (2010), la población total del partido es de 123.871 habitantes. La superficie total es de 493.500 hectáreas, organizadas administrativamente en doce cuarteles de los cuales once de ellos corresponden al espacio rural, por lo que las actividades primarias son 
predominantes en la zona bajo estudio. En cuanto a las características del medio físico, según lo expresado por Picone y Campo (2014), en el área de estudio predomina el clima templado continental debido al emplazamiento del partido en el Centro-Sudeste de la República Argentina, recibiendo la influencia del anticiclón del Atlántico Sur que aporta las masas de aire cálidas y húmedas y del anticiclón del Pacífico Sur que transporta aire frío y seco. La región se encuentra surcada por las sierras de Tandilia, que conforman un sistema serrano que se presenta en forma de arco, en sentido NO-SE, extendiéndose desde la ciudad de Olavarría hasta Mar del Plata (Ruiz de Galarreta et al., 2011). Ante tal diversidad de paisajes, en la ordenación ecológica del partido se distinguen tres grandes compartimentos, denominados serranías, Ilanura periserrana y llanura distal o deprimida (Sánchez et al., 1999). Caracterizados por poseer suelos del orden Molisol, predominando en gran parte el suborden argiudoles típicos con alto índice de productividad, esto permite que en el área de estudio seleccionada la agricultura se desarrolle e incremente de manera acelerada según los mercados globales, sin tener en cuenta las externalidades negativas. Tal como lo indican los trabajos de Vazquez et al. (2019) y Somoza et al. (2020) en el área bajo estudio se evidencia una fuerte tendencia hacia la agriculturización durante los últimos 30 años, por lo que se enfatiza en la necesidad de generar y fortalecer propuestas de gestión ambiental tendientes al desarrollo de agroecosistemas sostenibles. En cuanto a las características de los espacios rurales, según Nogar et al. (2017), se observan procesos de homogeneización productiva y el desplazamiento de productores, debido al creciente requerimiento de capital para lograr competitividad, la incorporación de innovaciones junto con la necesidad de nuevos conocimientos, el aumento en los precios de los arrendamientos y la presión ejercida por flujos extraterritoriales, que inducen la expulsión de actores.

Figura $\mathbf{N}^{\circ}$ 1. Ubicación del partido de Tandil en la provincia de Buenos Aires, Argentina

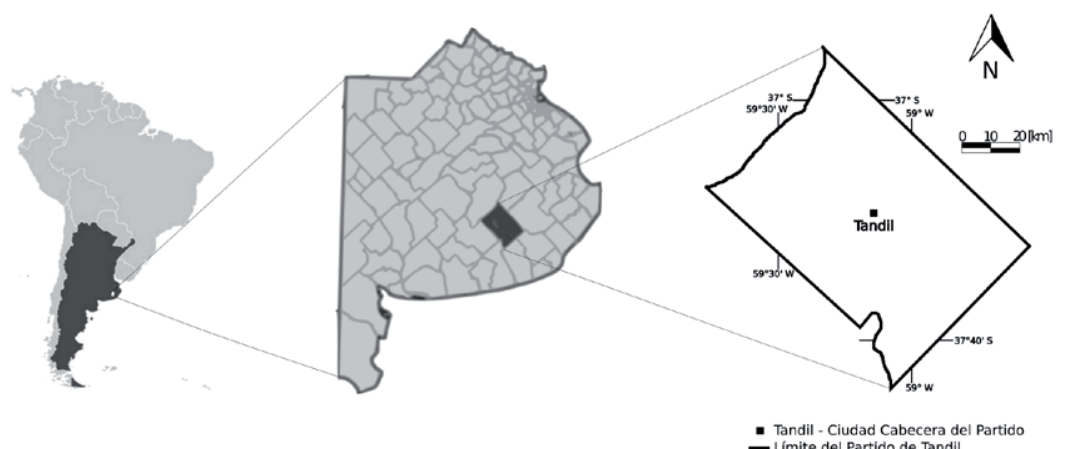

- Tandil - Ciudad Cabecera de

Elaboración propia. 
Para la identificación de los principales actores claves y la red de uso y comercialización de plaguicidas en el partido de Tandil, se llevaron a cabo reuniones informales con personas vinculadas a la actividad agrícola y se investigaron los principales organismos vinculados a esta actividad a partir de páginas web oficiales y bibliografía específica. Los actores claves se definen en este contexto, como personas, grupos u organizaciones que tienen interés en una temática en particular y pueden influenciar significativamente (positiva o negativamente) una intervención o son muy importantes para que una situación se manifieste de determinada forma (Tapella, 2007). Una vez definidos los actores claves, se realizaron entrevistas en profundidad a los mismos. Debido a la escasez de información o estudios previos sobre la temática en la zona, se decidió recurrir al uso de entrevistas basadas en un guion (Patton, 1990), las cuales tienen la particularidad de ofrecer libertad al entrevistador para ordenar y formular las preguntas, de acuerdo al curso de la conversación. Las mismas fueron dirigidas a informantes calificados de la cadena de uso y comercialización de plaguicidas del partido de Tandil, comprendiendo a referentes de las principales instituciones, organizaciones y privados.

Por otro lado, con el fin de conocer las prácticas de los productores agrícolas en relación a la manipulación y uso de los plaguicidas, se llevaron a cabo 20 encuestas estandarizadas. En cuanto al diseño muestral de la encuesta, se recurrió al método no probabilístico "de avalancha", "en bola de nieve" o "en cadena". De acuerdo a lo expresado por Blanco y Castro (2007), dicho muestreo consiste en solicitar a los encuestados que recomienden a posibles participantes, teniendo como ventaja una relación de confianza con los nuevos individuos a encuestar. Asimismo, al estar basado en referencias de pares en la actividad se obtiene cooperación de parte de los encuestados a la hora de contestar el cuestionario. Como desventaja se encuentra la posibilidad de conseguir una muestra reducida por verse truncada la red de contactos, presentando dificultades para establecer un criterio de representatividad de la población total. Las encuestas fueron utilizadas con el objetivo de relevar información vinculada a la última campaña de cultivo realizada por cada productor (tanto fina como gruesa), la cantidad de hectáreas sembradas, los plaguicidas utilizados en las mismas, detalles sobre cómo se lleva adelante la aplicación (los elementos y/o la maquinaria utilizada a tal fin, la tercerización de la actividad y el asesoramiento de un profesional idóneo), entre otras cuestiones. Finalmente, se buscó conocer la opinión de los encuestados respecto a una serie de afirmaciones vinculadas a temáticas ambientales generales. Para confeccionar estas afirmaciones, se tuvieron en cuenta 5 variables utilizadas para la definición del concepto de percepción ambiental (Borroto Pérez et al., 2011): visión ambiental (idea que tiene el 
individuo sobre la definición del concepto de ambiente y sus dimensiones); conocimiento de los problemas ambientales (identificación de alteraciones del ambiente en cualquiera de sus dimensiones); responsabilidad ambiental (apreciación que tienen los individuos sobre los agentes causantes de los problemas ambientales, así como los que deben intervenir para evitarla); actitud ambiental y decisión a incorporarse al cambio (predisposición del pensamiento humano a actuar a favor o en contra del entorno social, teniendo como base las vivencias, los conocimientos y los valores del individuo con respecto a su entorno) y formación medioambiental (apreciación que tienen los individuos sobre la calidad y efectividad de las acciones de educación ambiental que han recibido así como los modos y medios mediante los cuales la han adquirido). Es importante aclarar que no se pretendió construir el concepto de percepción de los productores agrícolas sobre el ambiente, sino conocer su opinión sobre algunos aspectos para comprender mejor su accionar. Finalmente, para la determinación de los principales plaguicidas utilizados y comercializados en el partido de Tandil, se utilizó la información proporcionada en las entrevistas a agronomías y a aplicadores, así como también las encuestas realizadas a productores agrícolas, recurriendo a la triangulación metodológica. Se entrevistó a la mayor cantidad de proveedores de agroinsumos y fumigadores posibles, con el fin de contrastar la información obtenida con los datos aportados por los productores encuestados.

\section{Actores claves involucrados en la red de uso y comercialización de plaguicidas en el partido de Tandil}

Se lograron identificar diez actores claves y sus interacciones agrupadas en relaciones de capacitación, control o comercial (Fig. 2). Asimismo, dichos actores fueron agrupados en 3 categorías principales: representantes de organismos gubernamentales, asociaciones civiles y privados. Entre los actores gubernamentales se identificó, en el nivel nacional, la Oficina Tandil del Servicio Nacional de Sanidad y Calidad Agroalimentaria (SENASA) y la Agencia de Extensión Rural Tandil del Instituto Nacional de Tecnología Agropecuaria (INTA), en el nivel provincial la Delegación Regional Fitosanitaria Zona V (perteneciente al Ministerio de Agroindustria), y localmente a las Direcciones de Medioambiente y de Asuntos Agropecuarios de la municipalidad de Tandil. Dentro de las asociaciones civiles se encuentran la Sociedad Rural Tandil, el Círculo de Ingenieros Agrónomos de Tandil (CIAT) y el Colegio de Ingenieros Agrónomos y Forestales de la provincia de Buenos Aires (CIAFBA). Por último, los privados identificados fueron los centros comercializadores de agroinsumos (agronomías), los aplicadores de plaguicidas tanto aéreos como terrestres y los productores agrícolas. 
Figura $\mathbf{N}^{\circ}$ 2. Red de actores claves vinculados al uso y comercialización de plaguicidas en el partido de Tandil (2019 - 2020)

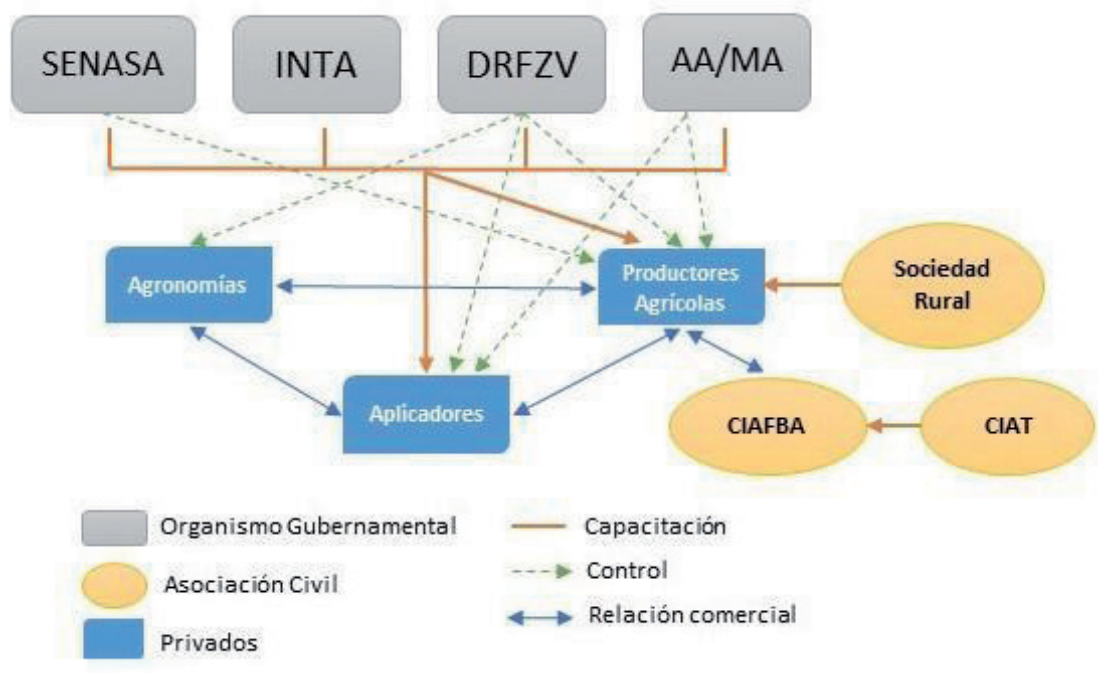

Fuente: elaboración propia.

A partir de las entrevistas, se destaca la falta de recursos y, por lo tanto, la falta de control sobre el uso y la comercialización de los plaguicidas utilizados en el partido. A nivel local, es preocupante la situación de la Delegación Regional Fitosanitaria Zona $\mathrm{V}$ ya que se trata del organismo encargado de fiscalizar el correcto cumplimiento de la Ley Provincial $N^{\circ}$ 10.699 y cuenta con graves carencias de recursos tanto humanos como económicos. Por otro lado, la escasa utilización de recetas agronómicas de aplicación en la práctica es un hecho afirmado por numerosos actores, entre ellos la trabajadora del INTA Tandil, la coordinadora de la Delegación Regional Fitosanitaria Zona V, el director de Asuntos Agropecuarios, la mitad de los locales que comercializan plaguicidas y los aplicadores terrestres consultados. Asimismo, según las entrevistas realizadas el destino de los envases vacíos de plaguicidas es diverso, como por ejemplo el acopio en un sector del campo, la incineración de los mismos y un comercio esporádico de plástico. Por otro lado, algunos de los actores claves entrevistados han puesto de relevancia una presión social creciente por parte de la sociedad, que exige un mayor control a la hora de utilizar plaguicidas. Asimismo, en cuanto a la posibilidad de producir alimentos sin recurrir a la utilización de dichos insumos químicos, es destacable que algunos de los actores claves han puesto en duda la posibilidad de producir alimentos bajo la modalidad de agroecología. Los entrevistados consideraron que se 
obtendrían menores rindes económicos y no se alcanzaría a lograr el abastecimiento necesario para alimentar a la población mundial. Por último, en cuanto a los aspectos positivos de la red de actores, se ha detectado una gran interacción interinstitucional entre los diferentes organismos gubernamentales debido a que participan conjuntamente a la hora de organizar las capacitaciones correspondientes, las cuales comprenden las jornadas de actualización obligatorias que indica el Decreto 499/91 reglamentario de la Ley Provincial $N^{\circ} 10.699$, y el taller destinado a extender el carnet habilitante para aplicar plaguicidas, que se trata de una jornada teórico práctica de un solo día de duración.

\section{Prácticas de los productores agrícolas en relación a la manipulación y uso de los plaguicidas}

Figura $\mathbf{N}^{\circ}$ 3. Respuestas de los productores agrícolas a las afirmaciones vinculadas al ambiente $(n=20)$.
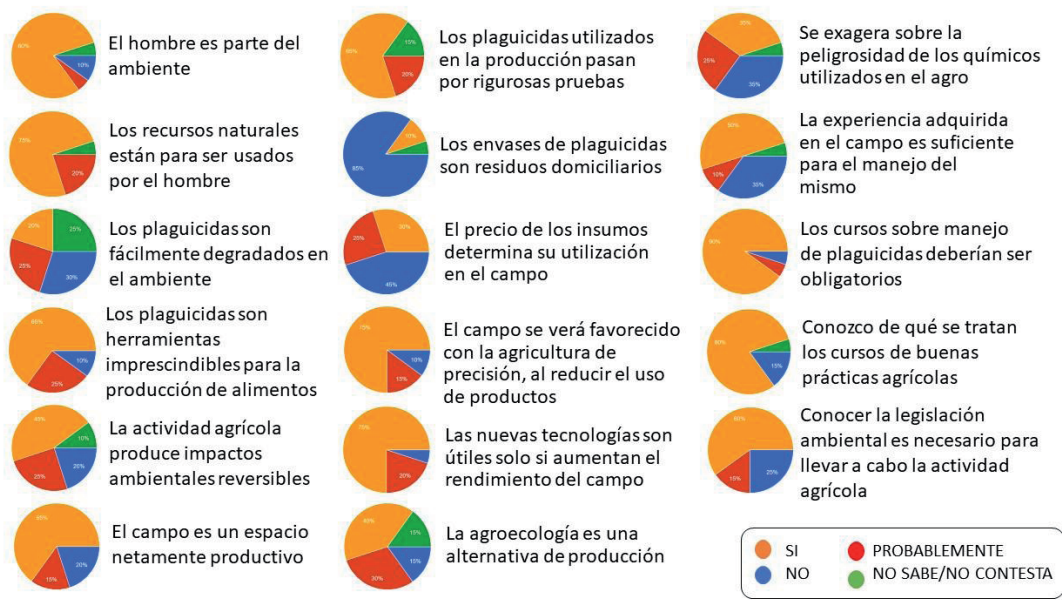

Fuente: elaboración propia

En cuanto a las prácticas en relación a la manipulación y uso de los plaguicidas, los 20 productores agrícolas encuestados son dueños o arrendatarios de campos que ocupan una superficie de 20.174 hectáreas (4\% de la superficie del partido de Tandil), con una superficie cultivada de 12.480 ha. El tamaño medio de las explotaciones encuestadas, es de 1.009 ha. Consultados por el cultivo que sembraron en la última campaña fina o de invierno, la mayoría de los encuestados respondieron trigo, cebada y avena. Al consultar sobre los herbicidas utilizados, se encuentran en primer lugar el glifosato, seguido por 2, 4 - D y atrazina. Dentro de los insecticidas 
se enumeran clorpirifos (como el mayormente utilizado) y cipermetrina. Por último, dentro de los fungicidas se nombró la utilización de clorotalonil. En cuanto a la siembra realizada en la última campaña gruesa o de verano, la mayoría de los encuestados afirmó haber cultivado soja, maíz, girasol y papa. Los herbicidas e insecticidas utilizados son los mismos que los empleados durante la campaña de fina o invierno, mientras que el fungicida aplicado fue mancozeb. Consultados sobre quién lleva a cabo la aplicación de los productos, el 55\% de los productores agrícolas aseguró que terceriza la actividad a una empresa fumigadora. Por otro lado, el $90 \%$ de los encuestados utiliza mosquitos (maquinaria utilizada para la aplicación de insumos químicos en la actividad agrícola), y el 85\% afirmó ser asesorado por un ingeniero agrónomo a la hora de tomar decisiones. En relación a las afirmaciones generales sobre el ambiente, los resultados obtenidos se detallan en la Figura $\mathrm{N}^{0} 3$.

\section{Principales plaguicidas utilizados y comercializados en el partido de Tandil}

En cuanto a los plaguicidas comercializados y utilizados en Tandil, tanto las entrevistas a las agronomías y aplicadores como las encuestas realizadas a productores agrícolas indican que en primer lugar se destacan los herbicidas (liderando ampliamente con el glifosato, seguido por el 2,4-D, dicamba y atrazina), en segundo lugar los insecticidas (con clorpirifos como el más usado y comercializado, seguido por la cipermetrina y lambdacialotrina), y por último los fungicidas (entre los que se destacan los triazoles y estrobilurinas como los productos más vendidos según las agronomías entrevistadas). Asimismo, mientras la venta de herbicidas se produce a lo largo de todo el año, en el caso de los insecticidas y fungicidas la demanda es más esporádica, debido a que la misma dependerá del momento en el año en el que se generen las condiciones propicias para que surja determinado hongo o insecto. Por otro lado, las agronomías entrevistadas indicaron un notable incremento de insumos banda verde en el mercado a lo largo de los últimos años, la sectorización de la venta ligada a las campañas fina o gruesa, la inexistencia de un límite para la compra de productos sin importar su clase toxicológica, y la posibilidad de adquirir plaguicidas mediante plataformas de E-Commerce, tales como Mercado Libre (https://www.mercadolibre.com.ar/). Debe hacerse hincapié en que todas las agronomías entrevistadas coincidieron en que el producto con mayor demanda es el glifosato, asegurando que al ser el más conocido la sociedad en general lo ha juzgado de peligroso erróneamente. Según los entrevistados, este producto es inocuo debido a que se trata de un producto banda verde según su clasificación toxicológica. Por otro lado, algu- 
nos de los productores encuestados declararon que utilizan tres productos prohibidos en el país: DDT, heptacloro y endosulfan. Sobre el total de 20 encuestados, el DDT fue mencionado por uno de los productores agrícolas, quien alegó utilizar este insumo tanto en la campaña de fina como la de gruesa. En cuanto al heptacloro, fue señalado por otro encuestado, quien especificó emplearlo durante la campaña de fina. El endosulfán fue nombrado por un productor dentro de los químicos utilizados en la campaña de gruesa. Por último, debe destacarse que los aplicadores terrestres entrevistados establecieron una relación entre el olor y la toxicidad de los insumos utilizados.

\section{Discusión}

Los actores clave consultados coincidieron en que los principales problemas asociados al uso y comercialización de plaguicidas en el partido de Tandil son: la escasez de recursos asignados al control, la baja utilización de la receta agronómica y el diverso destino final de los bidones vacíos. En relación a la escasez de recursos asignados a la problemática ambiental de los plaguicidas, es importante resaltar la falta de una ley nacional de presupuestos mínimos que sirva de marco referencial para que en ella se apoyen las legislaciones provinciales y se puedan aumentar las exigencias proteccionistas (Pórfido et al., 2014). Sin esta legislación marco, es muy difícil pensar en el uso de plaguicidas como una problemática que debe ser atendida como política de estado y, por lo tanto, a la cual se le asignen recursos económicos para la conservación de la salud ambiental y de las poblaciones humanas. En cuanto a la escasa utilización de la receta agronómica en la región, Martens (2012) realizó una investigación con 12 aplicadores del partido de Tandil, a fin de interpretar las características de la actividad en la región, en la que concluye que ninguno poseía receta profesional, sino que en algunos casos disponían de indicaciones escritas sin firma o se basaban en las instrucciones del marbete del producto. En un trabajo más actual, la misma autora realizó encuestas a participantes de una jornada de actualización obligatoria llevada a cabo por INTA para fumigadores que trabajan en el partido de Tandil, concluyendo que el uso de la receta de aplicación es muy poco frecuente (Martens, 2019). En la misma línea, Casadinho (2009) asegura que las restricciones en la comercialización de plaguicidas son menores, ya que es posible adquirir dichos productos en locales variados tales como ferreterías, forrajerías, semillerías, casa de venta de artículos de limpieza, sin la exigencia de una receta. Finalmente, se debe destacar que de acuerdo a lo expresado por el artículo 8 de la Ley Provincial № 10699 se encuentra prohibida la venta directa al usuario y/o aplicación de productos plaguicidas sin receta 
agronómica obligatoria confeccionada por un asesor técnico profesional ingeniero agrónomo u otro título habilitante matriculado en el Consejo Profesional de jurisdicción provincial.

Respecto a la gestión de bidones vacíos de plaguicidas, de las entrevistas realizadas se concluye que el destino de los envases es diverso. No se debe pasar por alto que los mismos se encuentran reglamentados por la Ley provincial $N^{0} 11.720$ de residuos especiales, bajo la categoría Y4 (desechos resultantes de la producción, la preparación y utilización de biocidas y productos fitosanitarios). Según la mencionada normativa, todo generador de residuos especiales es responsable del daño producido por los mismos, debiendo adoptar medidas paulatinas tendientes a disminuir la cantidad generada. Más recientemente, la Ley $N^{\circ} 27.279$ de presupuestos mínimos de protección ambiental para la gestión de los envases vacíos de fitosanitarios, introduce el concepto de "responsabilidad extendida y compartida" con los restantes eslabones de la cadena de gestión. Asimismo, prohíbe el abandono, vertido, quema o enterramiento de envases vacíos, así como la comercialización o entrega de los mismos a personas por fuera del sistema autorizado por la ley. El municipio de Tandil (2012) elaboró un documento denominado "Plan Estratégico Agroindustrial de Tandil 20122022 ", en el que se detallan lineamientos base enumerando una serie de proyectos prioritarios, dentro de los cuales se encuentra el reciclado y transformación de desechos plásticos provenientes del sector agrícola. En consecuencia, recientemente (04/01/2021), fue inaugurado un Centro de Acopio Transitorio (CAT) en colaboración con la empresa privada Reciplast, con quien hasta ese momento el gobierno municipal coordinaba jornadas abiertas anuales en las que se instaba a los productores agrícolas de la zona a acercar sus bidones vacíos. En dichas jornadas se disponía de un camión, el cual luego de completar su capacidad, transportaba los residuos hacia una sede vecina de la firma ubicada en la localidad de Benito Juárez (destino final). Por otro lado, algunos de los actores claves han discutido la posibilidad de producir bajo la modalidad de agroecología. Aunque existen numerosas experiencias agroecológicas en el país, es destacable el caso del establecimiento "La Aurora" ubicado en la localidad de Benito Juárez (a 80 kilómetros de Tandil). Se trata de un establecimiento familiar de 650 hectáreas que lleva a cabo una producción bajo el enfoque agroecológico, desde hace más de 15 años. Debido a su éxito económico y la durabilidad en el tiempo de esta experiencia, con frecuencia recibe visitas de personas que buscan interiorizarse sobre este modelo productivo (Carrasco et al., 2014; Palmisano, 2018). En cuanto a los resultados económicos logrados al implementar una producción agroecológica, Cerdá y Sarandón (2015) analizaron los rendimientos del cultivo de trigo durante una década (años 2000 al 2010), comparado los mismos con el promedio 
obtenido en campos de la zona. Como resultado, "La Aurora" obtuvo solo $200 \mathrm{Kg} / \mathrm{Ha}$ menos que los lotes vecinos que aplican el modelo tradicional de producción, logrando a pesar de esto una mayor ganancia bruta al omitir los gastos correspondientes a insumos químicos.

La mayoría de los productores agrícolas del partido de Tandil encuestados, afirmaron tercerizar la aplicación de plaguicidas, recurriendo principalmente a la maquinaria mosquito, siendo asesorados por un profesional. Actualmente, en el sistema agroalimentario existe una tendencia a la tercerización de las actividades productivas vinculadas a las tareas de siembra, fertilización, pulverización y cosecha, siendo cada vez más frecuente la contratación de servicios ofrecidos por terceros (Amador y Armelin, 2008; Cáceres, 2015; Cattaneo y Bocchicchio, 2019). En cuanto a la aplicación de plaguicidas, según lo expresado por Magdalena et. al. (2010), se trata de un proceso ineficiente debido a que solo un escaso porcentaje del químico entra en contacto con las plagas. Esto se debe a que durante el período que se encuentra entre la preparación del producto y su acción propiamente dicha, ocurren pérdidas que provocan efectos negativos tanto sobre la salud humana como el ambiente. Específicamente durante la etapa de pulverización, de acuerdo a los autores, se produce la intercepción por organismos no blancos, así como la deriva y la volatilización del producto. Una vez liberados al ambiente, los plaguicidas pueden llegar al aire, tierra, y al agua superficial y subterránea, contribuyendo a la degradación del medio y de los ecosistemas (Colombo y Sarandón, 2015). A esta contaminación, dada por el propio uso de los plaguicidas, se le suma la producida por el lavado inadecuado de tanques contenedores, las pérdidas en los depósitos de almacenamiento, los derrames accidentales, debido a la errónea disposición de los envases vacíos y su uso inadecuado por parte de la población, entre otras prácticas (del Puerto Rodríguez et al., 2014). En cuanto a los problemas que el uso de plaguicidas puede desencadenar, se encuentra la eliminación de organismos que no son objetivo de las aplicaciones (denominados organismos "no blanco"), la contaminación de ecosistemas, la aparición de nuevos brotes de plagas y la generación resistencia en ciertas poblaciones (Schaaf, 2013; del Puerto Rodríguez et al., 2014). Finalmente, la contaminación ambiental afecta directamente la salud del hombre. Las consecuencias de la exposición a plaguicidas para la salud humana dependen de numerosos factores, entre ellos, el tipo de plaguicida y su toxicidad, la dosis de exposición, la duración, el momento y las circunstancias en las que se los utiliza (SAyDS, 2007). En cuanto a los efectos concretos de intoxicaciones agudas, se encuentran numerosos síntomas, entre ellos temblores, cefaleas, mareos, convulsiones, arritmias cardíacas, dificultad y depresión respiratoria, neumonía por aspiración, asma, alergias, insuficiencia renal aguda, edema pulmonar, 
vómitos, mareos, lagrimeo, visión borrosa, incoordinación, sudoración, calambres, neuropatías, dolores musculares, atrofias musculares y parálisis (Verzeñassi, 2014). Por otro lado, respecto a las enfermedades crónicas producidas por estos químicos, se pueden enumerar diversos tipos de cáncer (Bassil et al., 2007), así como el aumento en la predisposición de efectos neurológicos, reproductivos y genotóxicos (Sanborn et al., 2007).

Nogar et al. (2017), aseveran que las relaciones socio productivas en los espacios rurales del partido de Tandil se ven redefinidas, ante el posicionamiento de redes de poder guiadas por la búsqueda de maximización productiva. Dicha eficiencia se sustenta en un incremento de los riesgos ambientales, vinculados a las formas de manejo de las unidades productivas. En esta línea, teniendo en cuenta las opiniones de los productores agrícolas encuestados, se pone de relevancia una visión utilitarista de la naturaleza, otorgando prioridad a los intereses económicos inmediatos por sobre la preocupación por las consecuencias sociales y ambientales a largo plazo (Matteucci 1998). Esta perspectiva antropocéntrica sitúa el énfasis en el logro de una mayor eficiencia en el proceso productivo y en el aumento de los ingresos monetarios obtenidos gracias al mismo (Toledo López, 2010). Al respecto, Andrade et al. (2017) aseguran que el modelo actual de agricultura industrial no debe limitarse a una concepción productivista de la realidad, sino que deberían tenerse en cuenta también las externalidades tanto ambientales como sociales resultantes, a fin de alcanzar una producción sostenible. Esto sumado al hecho de que algunos de los productores aseguraron recurrir a la agronomía de confianza a la hora de decidir la utilización de un producto. Esto pone de relevancia el rol secundario del asesor profesional en las actividades agrícolas necesarias para cada locación. Aquí se detecta un punto crítico, ya que el profesional que se desempeña como asesor comercial no visita el lote al momento de señalar la utilización de un plaguicida, sino que recomienda en base a lo que el productor le indica.

En referencia a los principales plaguicidas utilizados y comercializados en el partido de Tandil, los resultados obtenidos en el marco de la presente investigación concuerdan con lo expresado por Montedoro y Butinof (2019), quienes indican que en el mercado argentino de plaguicidas los herbicidas representan el mayor segmento en cuanto a la importancia de su facturación (encabezado por el glifosato como el principal principio activo comercializado), la segunda categoría corresponde a los insecticidas (liderada por clorpirifos como el producto más aplicado) y en tercer lugar se encuentran los fungicidas. Debe destacarse que, en las entrevistas realizadas a las agronomías, se menciona la inocuidad del herbicida glifosato, o la creencia de que se trata de un producto de baja peligrosidad al ser banda verde. Al respecto, Mitidieri y Corbino (2012) expresan que no 
existen plaguicidas inocuos ya que todos los productos de uso fitosanitario en la actividad agrícola presentan toxicidad, y por lo tanto, algún grado de peligrosidad. Sumado a esto, numerosas investigaciones confirman los efectos nocivos del glifosato sobre la salud humana (Paz y Miño et al., 2007; Benachour y Séralini, 2009; Gasnier et al., 2009). Por otro lado, se debe destacar que, entre los plaguicidas utilizados por los productores encuestados, se encuentran tres insecticidas prohibidos en el país: DDT (diclorodifeniltricloroetano), heptacloro y endosulfan. En el caso de los dos primeros, la legislación data de la década del 90 (Decreto 2121/90 y la Resolución 1030/92 de la Secretaría de Agricultura, Ganadería y Pesca respectivamente), mientras que el endosulfan se prohibió a partir de la fecha $1^{\circ}$ de julio de 2013 mediante la Resolución SAGPyA 511/11. De acuerdo al Instituto Regional de Estudios en Sustancias Tóxicas de la Universidad Nacional de Costa Rica, dentro de los efectos crónicos por exposición al DDT se encuentra su acumulación en tejidos, principalmente el graso, y su excreta a través de la leche humana, atravesando la membrana placentaria. Asimismo, fue catalogado como posible carcinógeno en humanos por el Centro Internacional de Investigaciones sobre el Cáncer. El heptacloro es hepatotóxico y nefrotóxico, produciendo depresión de glóbulos rojos en animales de experimentación. A largo plazo el endosulfan produce pérdida de memoria y daño cerebral difuso, en animales se ha reportado ceguera la cual se revierte al cesar la exposición (IRET-UNA, 2021). Cabe destacar que Colombo y Sarandón (2015) realizaron un relevamiento de la utilización de agroquímicos en la provincia de Buenos Aires, concluyendo que se siguen empleando químicos prohibidos desde hace más de 25 años en el país, tales como el DDT, Dieldrin y Endosulfán. Finalmente, es preocupante la relación que hicieron los aplicadores terrestres entrevistados entre el olor y la toxicidad de los insumos. Al respecto, Machado et al. (2012) realizaron una investigación en el cinturón verde de la ciudad de Córdoba en la cual concluyeron que existe la percepción de que los químicos utilizados actualmente son menos peligrosos que aquellos que utilizaban las generaciones anteriores, idea reforzada por el hecho de que los abuelos de los entrevistados no utilizaban ningún tipo de protección personal para llevar a cabo la aplicación.

\section{Conclusiones}

Las externalidades producidas por la agricultura tradicional se imponen como un punto de inflexión a partir del cual se comienza a repensar este modelo productivo dependiente de insumos actualmente cuestionado por la sociedad. Los efectos de los plaguicidas sobre el ambiente y por ende, sobre la población en general, implican la necesidad de realizar cambios. 
Consideramos fundamental ampliar la red de actores, incorporando a los trabajadores rurales que no son los propietarios o arrendatarios de los lotes en los que se desempeñan, organizaciones vecinales o ambientalistas, médicos especialistas en problemáticas derivadas de la exposición a estos compuestos, poblaciones rurales que residen en cercanía a las áreas fumigadas, académicos referentes en la temática, universidades públicas como entes generadores de conocimiento, consumidores de productos derivados del agro, entre otros. Por otro lado, es menester la sanción de una ley nacional marco que regule el uso y la comercialización de plaguicidas, contemplando presupuestos mínimos de protección ambiental, posibilitando la asignación del presupuesto necesario para que los organismos de control realicen su trabajo eficientemente según la normativa vigente. Sumado a esto, es imprescindible contar con información oficial y actualizada de la cantidad de plaguicidas utilizados con el fin de evaluar, no sólo la efectividad de los mismos en la producción actual, sino también el impacto ambiental producto de su uso. A pesar de que la receta agronómica digital podría ser una herramienta que permitiría visualizar e informatizar la cantidad de plaguicidas aplicados, a partir de las entrevistas realizadas se comprobó la escasa utilización de la misma. Asimismo, a nivel local se detecta una diversidad de destinos finales inadecuados de los envases vacíos de plaguicidas, por lo que es menester lograr un mayor control y seguimiento de los mismos. En cuanto a la habilitación para aplicar plaguicidas, se concluye que una capacitación de un solo día de duración no es suficiente, por lo que proponemos una jornada más extendida que incluya nociones básicas acerca de los posibles impactos que provocan las actividades agrícolas en el ambiente, y un acercamiento hacia modelos alternativos de producción de alimentos. Finalmente, el uso de plaguicidas debería reducirse paulatinamente, recurriendo a ellos luego de haber intentado mitigar las plagas presentes mediante otras alternativas, haciendo énfasis en los principios agroecológicos, en pos de lograr sistemas agroalimentarios sustentables cada vez más independientes de insumos externos y respetuosos con el ambiente.

\section{Referencias}

Albaladejo, C., Petrantonio, M., \& Iscaro, M. (2013). Transformaciones en los territorios rurales a partir del avance de la racionalidad productiva del agronegocio. Los pueblos del sudeste bonaerense en el marco de un nuevo contexto productivo sectorial. VIII Jornadas Interdisciplinarias de estudios agrarios y agroindustriales.
Amador, A., \& Armelin, J. M. (2008). Globalización e integración regional: su impacto en la prevención de los riesgos laborales en la agricultura de los países latinoamericanos. Revista de la Facultad de Agronomía, Universidad de Buenos Aires, 28(1), 43-51. Disponible en: http://ri.agro.uba.ar/files/download/ revista/facultadagronomia/2008Amador.pdf 
Andrade, F. H., Taboada, M. A., Lema, R. D., Maceira, N. O., Echeverria, H. E., Posse Beaulieu, G., ... \& Gamundi, J. C. (2017). Los desafíos de la agricultura argentina: satisfacer las futuras demandas y reducir el impacto ambiental. Ediciones INTA.

Bassil, K. L., Vakil, C., Sanborn, M., Cole, D. C., Kaur, J. S., \& Kerr, K. J. (2007). Cancer health effects of pesticides: systematic review. Canadian Family Physician, 53(10), 1704-1711.

Benachour, N., \& Séralini, G. E. (2009). Glyphosate formulations induce apoptosis and necrosis in human umbilical, embryonic, and placental cells. Chemical research in toxicology, 22(1), 97-105. Disponible en: https://www.essencechem.com/product/ glyphosate.html

Blanco, M. C. M. C., \& Castro, A. B. S. (2007). El muestreo en la investigación cualitativa. Nure investigación, 27(4). Disponible en: https://www.nureinvestigacion. es/OJS/index.php/nure/article/view/340

Borroto Pérez, M., Pérez, L. R., Ramírez, A. R., \& Vázquez, B. A. L. (2011). Percepción ambiental en dos comunidades cubanas. $M$ $+A$, revista electrónica de medioambiente, (10), 13-29.

Cáceres, D. M. (2015). Tecnología agropecuaria y agronegocios. La lógica subyacente del modelo tecnológico dominante. Mundo agrario, 16(31). Disponible en: https:// www.mundoagrario.unlp.edu.ar/article/view/ MAv16n31a08

Carrasco, A. E., Sánchez, N. E., \& Tamagno, L. E. (2012). Modelo agrícola e impacto socioambiental en la Argentina: monocultivo y agronegocios. Series: Serie Monográfica Sociedad y Ambiente: Reflexiones para una nueva Latinoamérica; Monografía № 1 .

Carrasco, N., Cerdá, E., Zamora, M., \& Ferrín, M. G. (2014). El caso de "La Aurora": un ejemplo de aplicación del enfoque agroecológico en sistemas extensivos del sudeste de la Provincia de Buenos Aires, Benito Juárez, Argentina.

Casadinho, J. S. (2009). La problemática del uso de plaguicidas en Argentina. Modelos productivos e impacto en el ambiente.
In XXVII Congreso de la Asociación Latinoamericana de Sociología. VIII Jornadas de Sociología de la Universidad de Buenos Aires. Asociación Latinoamericana de Sociología.

CASAFE - Cámara de Sanidad Agropecuaria y Fertilizantes. (2016). Mercado Argentino de Productos Fitosanitarios, año 2016. Argentina, Buenos Aires. Disponible en: https://www.casafe.org/publicaciones/datosdel-mercado-argentino-de-fitosanitarios/

Cattaneo, C. A., \& Bocchicchio, A. M. (2019). Dinámica socio organizacional en el sistema agroalimentario. Revista mexicana de sociología, 81(1), 7-35. Disponible en: https://www. researchgate.net/publication/330026209 Dinamica_sociorganizacional_En_el_sistema_agroalimentario

Ceccon, E. (2008). La revolución verde: tragedia en dos actos. Ciencias, 91(091). Disponible en: https://www.redalyc.org/ pdf/644/64411463004.pdf

Cerdá, E., \& Sarandon, S. J. (2015). Producción extensiva de cereales y carne bovina en forma agroecológica: El caso "La Aurora", sudeste de la Prov. de Buenos Aires, Benito Juárez, Argentina. Congreso Latinoamericano de Agroecología-SOCLA (7 al 9 de octubre de 2015, La Plata).

Colombo, C., \& Sarandón, S. (2015). Relevamiento de la utilización de agroquímicos en la provincia de Buenos Aires. Mapa de situación e incidencia sobre la salud. Defensor del pueblo, Provincia de Buenos Aires. Universidad Nacional de La Plata. 532pp.

del Puerto Rodríguez, A. M., Suárez Tamayo, S., \& Palacio Estrada, D. E. (2014). Efectos de los plaguicidas sobre el ambiente y la salud. Revista Cubana de Higiene y Epidemiología, 52(3), 372-387.

Gasnier, C., Dumont, C., Benachour, N., Clair, E., Chagnon, M. C., \& Séralini, G. E. (2009). Glyphosate-based herbicides are toxic and endocrine disruptors in human cell lines. Toxicology, 262(3), 184-191.

IRET-UNA - Instituto Regional de Estudios en Sustancias Tóxicas. (2021). Manual de plaguicidas de Centroamérica. Universidad Nacional de Costa Rica. Disponible en: 
http://www. plaguicidasdecentroamerica. una.ac.cr

Machado, A. L., Ruiz, M. V., Sastre, M. A., Butinof, M., Blanco, M., Lantieri, M. J., ... \& Díaz, M. D. P. (2012). Exposición a plaguicidas, cuidado de la salud y subjetividad. Kairos: Revista de temas sociales, (30), 4-17.

Magdalena, J. C.; Fernández D.; Di Prinzio, A. \& Behmer, S. (2010). Tecnología de aplicación de agroquímicos. Capítulo 1. Programa Iberoamericano de Ciencia y Tecnología para el Desarrollo (CYTED). Red Temática 107RT0319: Técnicas sostenibles de distribución de plaguicidas.

Manuel-Navarrete, D., Gallopín, G. C., Blanco, M., Díaz-Zorita, M., Ferraro, D., Herzer, H., ... \& Celis, A. (2005). Análisis sistémico de la agriculturización en la pampa húmeda argentina y sus consecuencias en regiones extra pampeanas: sostenibilidad, brechas de conocimiento e integración de políticas. CEPAL.

Martens, F. (2012). Guía para el uso adecuado de plaguicidas y la correcta disposición de sus envases. Boletín de divulgación del INTA, (41), 22.

Martens, F. (2019). Una mirada de la aplicación de fitosanitarios desde "el mosquito". Visión Rural. Ediciones INTA. ISSN 03287009.

Matteucci, S. D. (1998). La creciente importancia de los estudios del medio ambiente. Disponible en: https://www.researchgate. net/publication/269222537

Mitidieri, M. S., \& Corbino, G. B. (2012). Manual de horticultura periurbana. Ediciones INTA.

Montedoro, F. D., \& Butinof, M. (2019). Percepción poblacional de riesgo de exposición a plaguicidas en una localidad de la Pampa Húmeda Argentina. Revista de Salud Ambiental, 19(2), 136-147.

Municipio de Tandil. (2012). Plan Estratégico Agroindustrial de Tandil 2012-2022. Disponible en: http://www.econ.unicen. edu.ar/extension/vinculacion/images/Informe\%20Final\%20Tandil\%2012-07\%20_final_\%20(2).pdf
Nogar, A. G.; Jacinto, G.; Nogar, M. L. (2017). "Redes de poder, deslocalización y cambios en los usos productivos rurales pampeanos". Revista de Estudios Territoriales Pampa. Universidad del Litoral. (15). 31-46. Disponible en: https://bibliotecavirtual.unl.edu.ar/publicaciones/index.php/ PAMPA/article/view/6601

Palmisano, T. (2018). Las agriculturas alternativas en el contexto del agronegocio. Experiencias en la provincia de Buenos Aires, Argentina. Estudios sociales (Hermosillo, Son.), 28(51), 0-0. Disponible en: https:// www.ciad.mx/estudiosociales/index.php/es/ article/view/513

Patton, M. Q. (1990): Qualitative Evaluation and Research Methods, London: Sage.

Paz y Miño, C., Sánchez, M. E., Arévalo, M., Muñoz, M. J., Witte, T., De-la-Carrera, G. O., \& Leone, P. E. (2007). Evaluation of DNA damage in an Ecuadorian population exposed to glyphosate. Genetics and Molecular Biology, 30(2), 456-460.

Picone, N., \& Campo, A. M. (2014). Comparación urbano-rural de parámetros meteorológicos en la ciudad de Tandil, Argentina.

Pordomingo, A. J. (2003). Gestión ambiental en el feedlot. Guía de buenas prácticas. INTA Anguil.

Pórfido, O. D., Butler, E., de Titto, E., Issaly, P., \& Benítez, R. (2014). Los plaguicidas en la República Argentina. Ministerio de Salud de la Nación, Argentina.

Rodríguez, J. (2012). Los cambios en la producción agrícola pampeana. El proceso de sojización y sus efectos. Voces en el Fénix, 3(12), 14-21.

Ruiz de Galarreta, A., Barranquero, R. S., Varni, M., \& Rodríguez, C. I. (2011). Geología e hidrolitología de la cuenca del Arroyo Langueyú, Provincia de Buenos Aires (Argentina). Ciencia, docencia y tecnología, 20(43), 157-176.

Sanborn, M., Kerr, K. J., Sanin, L. H., Cole, D. C., Bassil, K. L., \& Vakil, C. (2007). Non-cancer health effects of pesticides: systematic review and implications for family 
doctors. Canadian family physician, 53(10), 1712-1720.

Sánchez, R., Mattus, G., \& Zulaica, L. (1999). Compartimentación ecológica y ambiental del partido de Tandil (Provincia de Buenos Aires). Congreso Ambiental 99, 338-346.

Sarandón, S. J., \& Flores, C. C. (2014). Agroecología: bases teóricas para el diseño y manejo de agroecosistemas sustentables. Editorial de la Universidad Nacional de La Plata (EDULP).

SAyDS - Secretaría de Ambiente y Desarrollo Sustentable. (2007). La problemática de los agroquímicos y sus envases, su incidencia en la salud de los trabajadores, la población expuesta y el ambiente. Secretaría de Ambiente y Desarrollo Sustentable: OPS: AAMMA. 1 a ed. Buenos Aires: 312 pp.

Schaaf, A. A. (2013). Uso de pesticidas y toxicidad: relevamiento en la zona agrícola de San Vicente, Santa Fe, Argentina. Revista mexicana de ciencias agrícolas, 4(2), 323-331.

Segrelles, J. A. (2001). Problemas ambientales, agricultura y globalización en América Latina.

Sili, M. (2005). La Argentina rural: de la crisis de la modernización agraria a la construcción de un nuevo paradigma de desarrollo de los territorios rurales. Ediciones INTA, Buenos Aires.

Somoza, A., Vazquez, P. S., Zulaica, M. L., \& Sacido, M. B. (2020). Dinámica espaciotemporal de usos del suelo en sistemas ecológicos de la región pampeana austral, Argentina: Agriculturización en el Partido de Tandil. Sin datos.

Tapella, E. (2007) El mapeo de Actores Claves, documento de trabajo del proyecto "Efectos de la biodiversidad funcional sobre procesos ecosistémicos, servicios ecosistémicos y sustentabilidad en las Américas: un abordaje interdisciplinario", Universidad Nacional de Córdoba, Inter-American Institute for Global Change Research (IAI).

Toledo López, V. (2010). Lenguajes de valoración y conflictos territoriales en el campo argentino: reflexiones en torno a la producción de agrocombustibles. Desenvo/vimento e Meio Ambiente, 22, 13-24. Disponible en: cademia.edu/32704933/2010_-Rev Desemvolvimento_e meio_amb_- VTT-Lenguajes_de_valoración_y_conflictos_territoriales_en_el_campo_argentino.pdf

Vazquez, P. S., \& Zulaica, M. L. (2013). Agriculturización e impactos ambientales desde 1988 a la actualidad, en la Cuenca Superior del Arroyo Langueyú y en el Partido de Tandil (Provincia de Buenos Aires). Revista científica digital EntreVistas. 1-13pp.

Vazquez, P. S., Zulaica, M. L. \& Somoza, A. (2019). Tasas de cambio de uso del suelo y agriculturización en el partido de Tandil, Argentina. Geoambiente on-line. nro. 34. 66-86.

Verzeñassi, D. (2014). Agroindustria, salud y soberanía. El modelo agrosojero y su impacto en nuestras vidas. La Patria Sojera. El modelo agrosojero en el Cono Sur. Ed. El Colectivo (Argentina), 31-48.

\section{Notas}

1 Este trabajo se enmarca en los resultados obtenidos en una tesis de grado, correspondiente a la Lic. en Diagnóstico y Gestión Ambiental, perteneciente a la Universidad Nacional del Centro de la Provincia de Buenos Aires.

2 Lic. en Diagnóstico y Gestión Ambiental.

3 Becaria Doctoral CONICET. Lic. en Tecnología Ambiental.

4 Docente e Investigadora Independiente CONICET.

5 Docente e Investigadora Adjunta CONICET. 